\title{
Long PFN nailing in comminuted high subtrochanteric fractures femur in lateral position on ordinary table
}

\author{
Shashikant B. Ganjale \\ Consultant Orthopaedics Surgeon, Dept. of Orthopaedics, Ashwini Sahakari Rugnalaya Ani Sanshodhan Kendra, Solapur, \\ Maharashtra, India
}

*Corresponding Author: Shashikant B. Ganjale

Email: sbganjale_live@rediffmail.com

\begin{abstract}
Introduction: Most of the hip fractures are managed in supine position on a fracture table with foot attached to foot plates and biplanar fluoroscopy supervision under c-arm. But this procedure has its own difficulties in managing especially comminuted subtrochanteric fractures, where in different fragments and segments are being pulled by strong muscles around hip, and getting good reduction and alignment is difficult due to displaced bony fragments being held in traction on fracture table and cannot be manipulated easily. This leads to prolonged operative time, opening of fracture site, increased bleeding, increased chances of infection and over all increased anaesthesia time adding up to risk factors. The purpose of this study was to report how these difficulties can be overcome by operating on a simple radioluscent top operating table in lateral position without a fracture table. Materials and Methods: 74 cases of subtrochanteric fractures with or without comminution were operated in lateral position on simple ordinary radioluscent top table with lesser attachments and adjustments to operating table during the study duration were included in the study. We used long Proximal Femur Nail (PFN) (length more than $25 \mathrm{cms} 34,36,38$, 40cms long) to fix these fractures.

Results: In the present study, age group of patients was $25 \mathrm{yrs}$ to $74 \mathrm{yrs}$. Male patients $(83.7 \%)$ were more than female patients (16.3\%) An average of less than $150 \mathrm{ml}$ in closed surgery. The average operative time for all cases was 80 minutes. Union time varied from 16 weeks in simple fractures to 26 weeks in comminuted fractures. Clinical function of hip and knee was excellent with full range of movements.

Conclusions: Reduction and fixation of proximal femoral fractures in the lateral position with fluoroscopy or portable radiography in the anteroposterior view for small set ups and rural hospitals that lack a fracture table or advanced fluoroscopic devices may be executable and probably safe.
\end{abstract}

Keywords: Long PFN, Subtrochanteric fracture, Lateral position, Ordinary operating table.

\section{Introduction}

The frequency of fractures in and around hip are increasing along with increased involvement of elderly osteoporotic patients and high incidence of high velocity trauma occurring in road traffic accidents leading to comminuted fractures. Intertrochanteric and subtrochanteric fractures make up of about $50 \%$ of hip fractures. ${ }^{1-3}$

There are different methods and implants for managing these fractures in different positions to enumerate a) supine position on fracture table b) In lateral position on ordinary table and c) prone position with extended posterolateral exposure. ${ }^{3-8}$

Kuntscher introduced lateral position for intramedullary nailing of femur shaft fractures in 1940, later Davis and Frymoyer described for management of intertrochanteric and subtrochanteric fractures with Jewett nail plate in $1969 .^{7}$

The main aim in any fracture is to achieve good reduction and alignment with good stable fixation with any implant. Majority of the hip fractures are operated on fracture table in supine position. But fixing the patient on the fracture table is difficult and timeconsuming. Positioning of obese patient on a fracture table and securing to footplates of fracture table is still more difficult because of pendulous abdomen and gluteal folds falling by the side. In the case of unsuccessful closed reduction, exposure of the fracture site for open reduction is difficult, because the patient is in the supine position and the hip is in traction while extended. Secondly operating surgeon finds some difficulty in the operative field to tackle the difficulty such as haemostasis, retraction of soft tissues etc. When there is associated ipsilateral fractures down below in leg and opposite limb fractures positioning a polytraumatized patient on fracture table becomes all the more difficult. ${ }^{7,9}$ The possibility of side effects of fracture table injuries like pudendal nerve neuropraxia perennial pressure sore cannot be ruled out. ${ }^{10,11}$

Connelly et al. had described that lateral decubitus position facilitated the reduction and exposure for locking plate fixation in ten proximal fractures of femur. ${ }^{9}$

Carr et al described the use of intra-operative imaging with C-arm to obtain excellent lateral view. ${ }^{12}$

Femoral intramedullary interlocking nailing in lateral position is described for fracture shaft femur, but long PFN in lateral position for complex comminuted subtrochanteric fracture is seldom done and found in literature. ${ }^{3,7-14}$ The purpose of this study was to demonstrate the technique of surgery using long PFN in complex comminuted subtrochanteric fractures in lateral position on an ordinary radiolucent top table without fracture table. 


\section{Materials and Methods}

74 cases of subtrochanteric fractures with or without comminution were operated in lateral position on simple ordinary radiolucent top table with lesser attachments and adjustments to operating table in our hospital during period from 2010 to 2017. Long PFN (length more than $25 \mathrm{cms} 34,36,38,40 \mathrm{cms}$ long) was used to fix these fractures. One case was operated using regular PFN (25cms long) due to unavailability of long PFN and could get excellent fixation and full bony union in the follow up.

The proforma was used to record like age, sex, fracture type, operative time, blood loss, quality of reduction and alignment, ease of screening under $\mathrm{C}$ arm in lateral position, and good clinical function at hip and knee.

Comminuted subtrochanteric complex and segmental fractures with medical risks were operated in institution, where as simple subtrochantric fractures were operated in smaller set up private orthopaedic hospitals. All the patients were investigated medically with respect to medical fitness and cardio respiratory fitness by taking help of our physician, cardiologists and anesthesiologists.

Majority of the cases were due to following road traffic accidents and some were due to fall from height. One case was pathological fracture.

Inclusion Criteria: Only simple and comminuted subtrochanteric fractures of femur were included.
Exclusion Criteria: Associated fractures and injuries and their management were excluded.

Gentle traction with relaxed muscles of thigh and hip in lateral position made our job of getting reduction and alignment easy. Cannulated rigid reamer was passed through entry proximally and Joy sticking maneuvering with cannulated reamer to negotiate the distal segment and passing the guide wire was easy procedure. In difficult situations, we used percutaneous unicortical schanz pin fixation just $5 \mathrm{cms}$ away fracture site in one or both segments and by joystick maneuvering we could get the reduction. In irreducible case, last resort was to open the fracture site.

\section{Operative Technique}

All patients were operated in lateral position on simple ordinary radioluscent top operating table. Patients were operated under regional or general anaesthesia as decided by anesthesiologist and general condition of patient.

The patient is placed in lateral position with fractured limb up. Two firm supporting bolsters are placed one anterior and one posterior and are well secured with Velcro straps to stabilize the patient in lateral position. One flat pillow is placed between two thighs to support and prevent sagging of distal segment. The pillow and lower limb is secured well with Velcro strap. The operative limb is free to move and adjust alignment or for giving traction for getting proper reduction (Fig. 1)
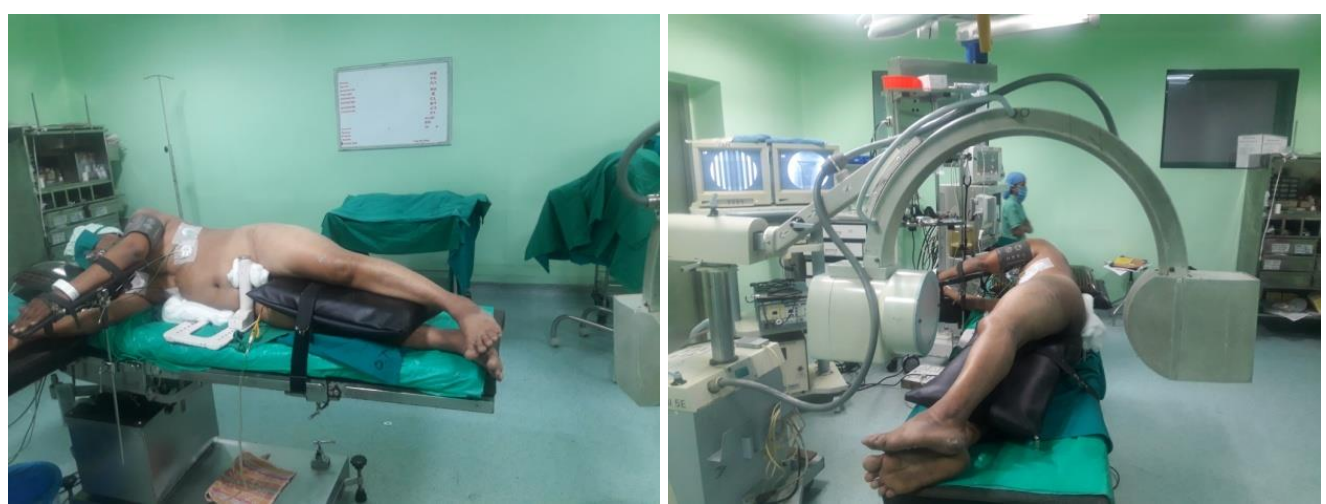

Fig. 1: Clinical photograph of lateral position of patient with fractured limb up and C arm adjustment

As the patient is in lateral position the c-arm is positioned with camera anterior and $\mathrm{x}$-ray tube posterior to visualize AP view with $\mathrm{C}$ arm coming from above or below. Operating surgeon is standing behind the patient to make entry in proximal femur and complete the procedure. (Fig. 2)

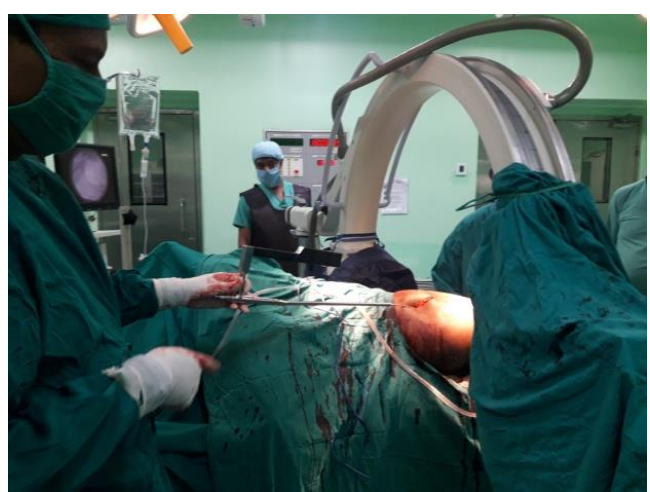

Fig. 2 
All tubing's like IV fluid tubings, airway tubings, and catheter are taken due care so that they are not kinked nor obstructing the operative field and screening of $\mathrm{c}$ arm view.
The whole fractured lower limb is scrubbed from umbilical region upto ankle level and prepped with povidine iodine. The fractured limb is freely draped so as to have free movements at hip and knee during the surgery. (Fig. 3A)
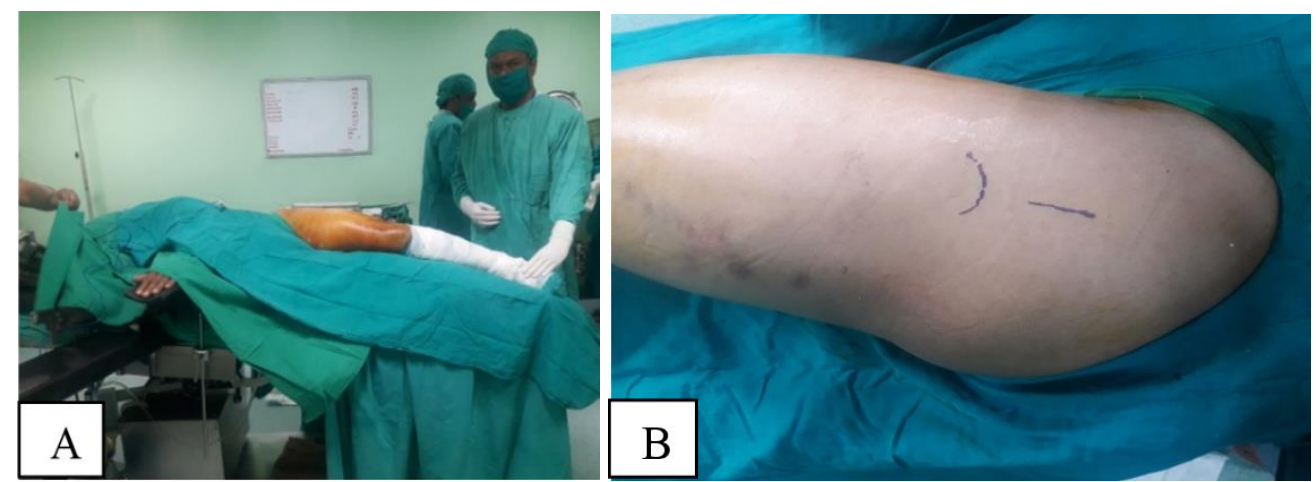

Fig. 3A, B: Full and free draping of fractured limb and incision marked

Primary screening of fractured limb is done under $\mathrm{C}$-arm to know the fracture geometry and check the reduction and alignments that can be achieved primarily and necessary adjustments be done accordingly. (Fig. 4)

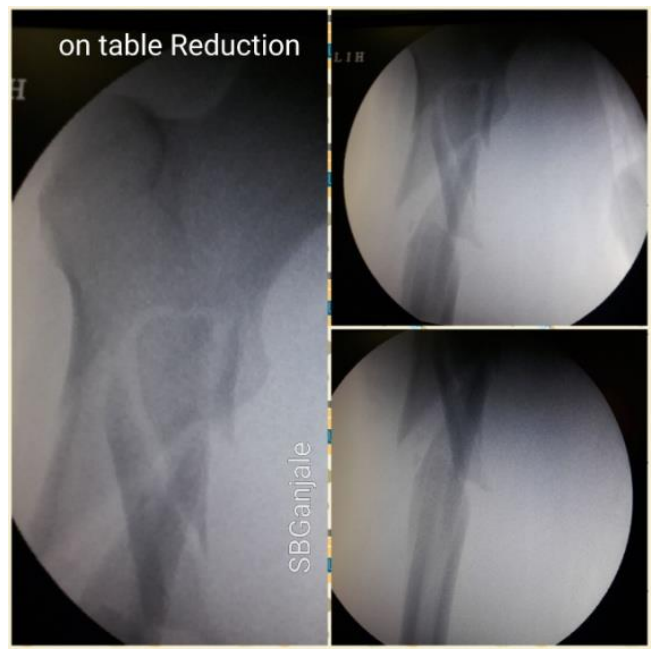

Fig 4. Screening of Fracture alignment in lateral position

Incision is marked over superolateral quadrant over gluteal region the incision is about $5 \mathrm{cms}$ proximal to tip of greater trochanter. (Fig. 3B) Tensor fascia lata and Gluteus maximus muscle is split vertically in the line of incision to reach pyriformiss fossa. Entry in pyriformis fossa is done with smaller size $6 \mathrm{~mm}$ straight $\mathrm{k}$-nail reamer, and later enlarged with larger diameter reamer. Now a guide wire is passed through the entry to negotiate the fracture site and distal segment. (Fig. 5)

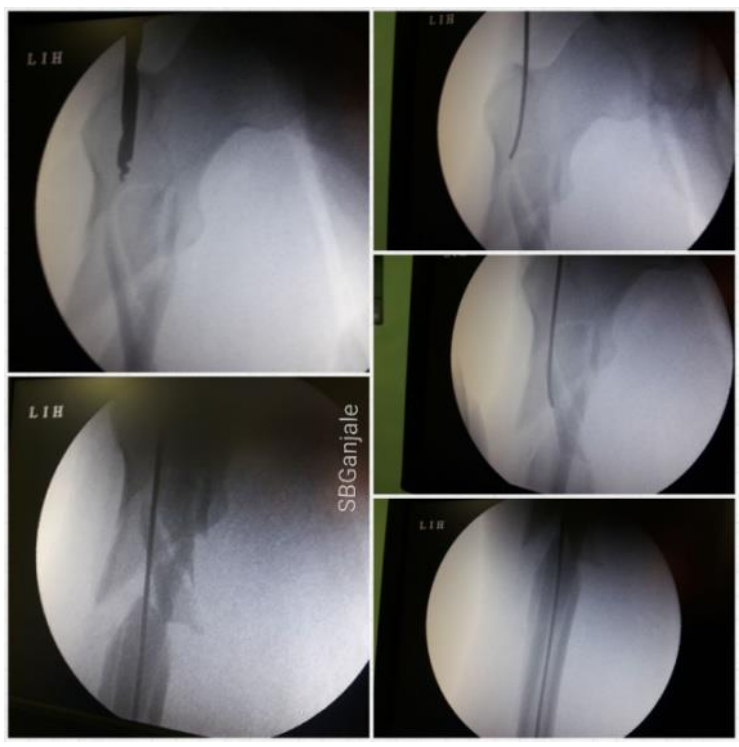

Fig. 5: Entry and passing guide wire

A minimal traction by an assistant is needed. Usually guide wire can be passed easily into distal segment if the fracture is not comminuted, but if there is comminution it becomes difficult as guide wire traverses, it goes in different directions according to geometry of fracture and displaced bony fragments. In this situation, we used rigid cannulated reamer threaded over guide wire upto the fracture site now this rigid cannulated reamer acts as a joystick to negotiate the fracture site to enter in distal segment. As the proximal segment is short joystick maneuvere can be easily done to get the distal segment reduction alignment and negotiate across fracture site into distal segment easily this is the crux and secret and advantage of getting reduction in lateral position as the muscles around the hip and thigh are relaxed and manipulation is easy.

Once the cannulated reamer is in the distal segment the guide wire is passed through the cannulated reamer 
into distal segment. A grating sensation is felt while passing of guide wire into distal segment. At this stage guide wire placement is checked in c arm. (Fig. 5)
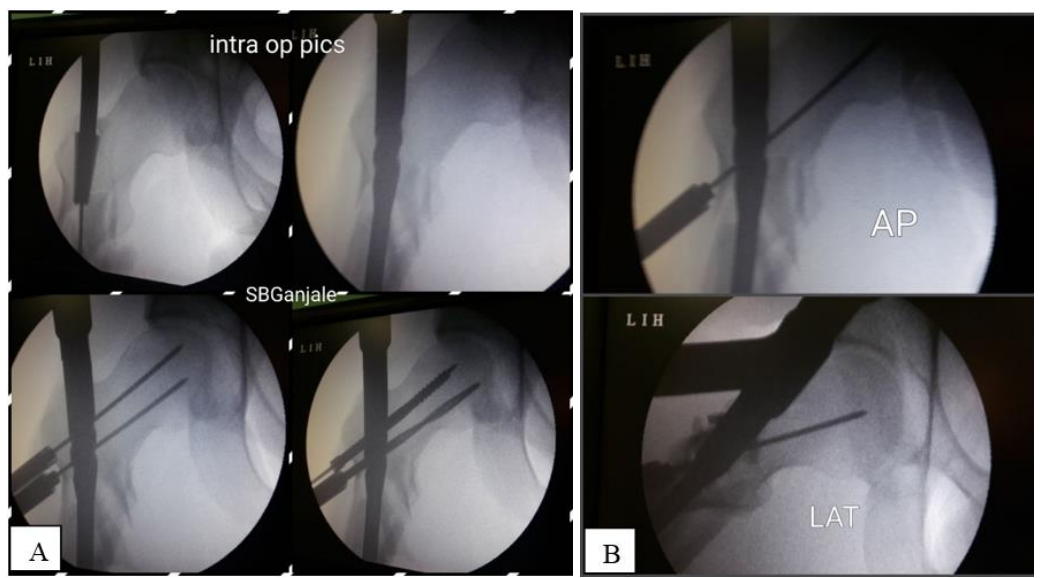

Fig. 6A: Enlarging entry with starting reamer and passing long PFN; 6B. Head neck screw holes aligned and GW passed in head and neck AP and Lat view

Further reaming can be done with rigid cannulated or flexible reamers. In comminuted fractures we preferred to ream gently and controlled reaming with rigid cannulated reamers. Reaming was done starting from $7 \mathrm{~mm}$ to $12 \mathrm{~mm}$ in the distal segment to fit thicker diameter nail. We reamed one $\mathrm{mm}$ more than the required nail diameter and passed one $\mathrm{mm}$ smaller diameter long PFN nail than what has reamed. Proximal portion of femur was reamed with starting reamer of long PFN to seat the proximal portion of long PFN. After thorough reaming long PFN of correct size was passed over guide wire, the proximal holes of Long PFN was aligned at the level of neck and head region of femur in AP view of $\mathrm{C}$-arm. (Fig. 6A, B) A required anteversion of neck was made by adjusting long PFN jig accordingly. Proximal guide wires were passed through jig sleeve in to neck and head. The position of these guide wires were checked under $\mathrm{c}$ arm in AP and lateral views

Now viewing of guide wires in neck and head in $\mathrm{AP}$ view in $\mathrm{C}$-arm is easy. To visualize lateral view of neck, the thigh is gently flexed, abducted and externally

rotated. This gives a lateral view of neck portion and placement of guide wires in neck and head portion. (Fig. 7A, B)

The placement of guide wire should be central or posterocentral.

Once placement of guide wires is confirmed the head neck screws are fixed. Rotational alignment of fracture is assessed by checking patella is facing parallel to floor in neutral position and the knee is flexed more than 90 degree.

Word of caution is that, the patient should be in fixed lateral position during all the procedure. At times during manipulation traction to get reduction makes the supporting bolsters loose and patient position becomes semiprone or semi supine, in this position the images in $\mathrm{C}$-arm though appears correct, the lengths of head neck screws may get altered due to magnification and the screw length may be seen bigger in length in post operative X-ray.

Distal interlocking of PFN is done by freehand technique by identifying interlocking holes and interlocks it under $\mathrm{C}$-arm.
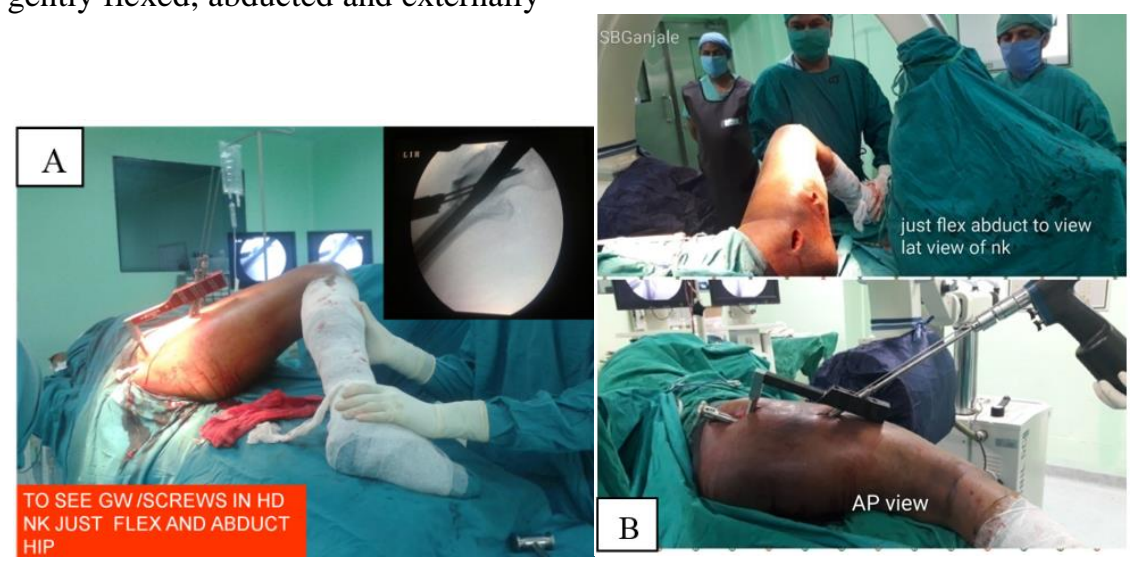

Fig. 7A, B: Clinical photo passing head neck guide wire and reaming and to show how lateral view of neck is seen 


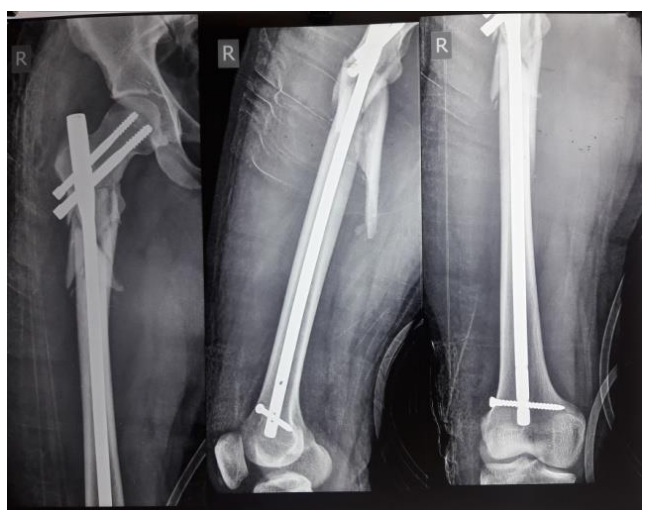

Fig. 8: Immediate post op X-ray

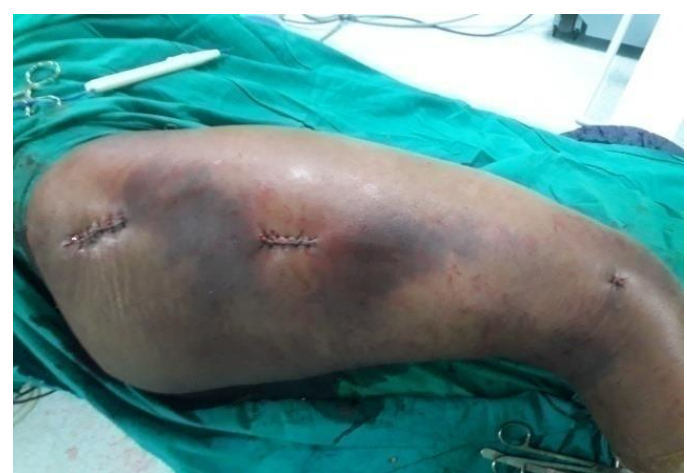

Fig. 9: Clinical photo skin incision

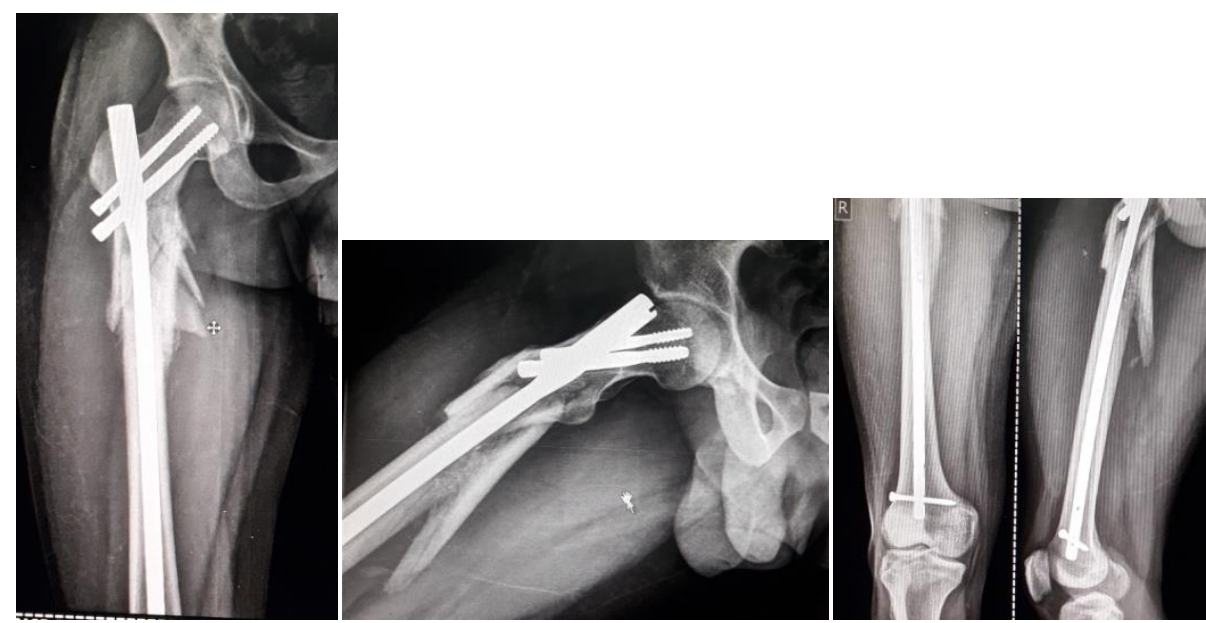

Follow up at 2 months fracture consolidation

Operative wound wash is given with normal saline and betadine and incisions are closed. Pressure bandage is applied to whole lower limb from foot to proximal thigh.

IV antibiotics for five days and oral antibiotics for five days were given post operatively. Dressings were done on third seventh and tenth day. Bed side quadriceps exercises were taught and done. Patient was ambulated non weight bearing on walker as per tolerance of patient after all tubings like catheter and IV fluids were out. Patients were discharged on fifth to seventh day. Pain was controlled with oral analgesics Suture removal was done on 10 to $12^{\text {th }}$ post operative day. They were advised to follow up every six weeks to assess them clinically radiologically and function. Knee flexion exercises and Quadriceps strengthening exercises were taught in immediate post operative period was continued to achieve maintain good function. Patients were instructed not to sit cross legged in bed and squatting in post operative period as cross legged sitting and squatting causes twisting movement of hip and undue stress on implant mainly head neck screws leading to loosening and back out.

\section{Clinical Scenario}

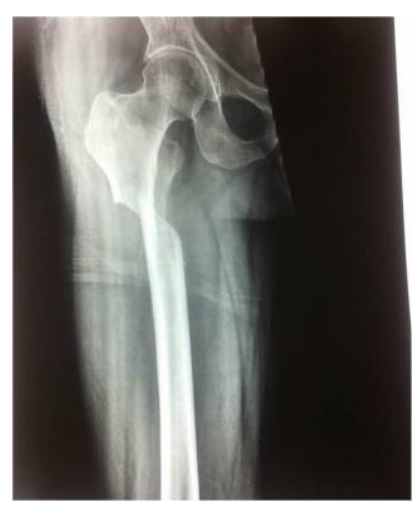

Pre op

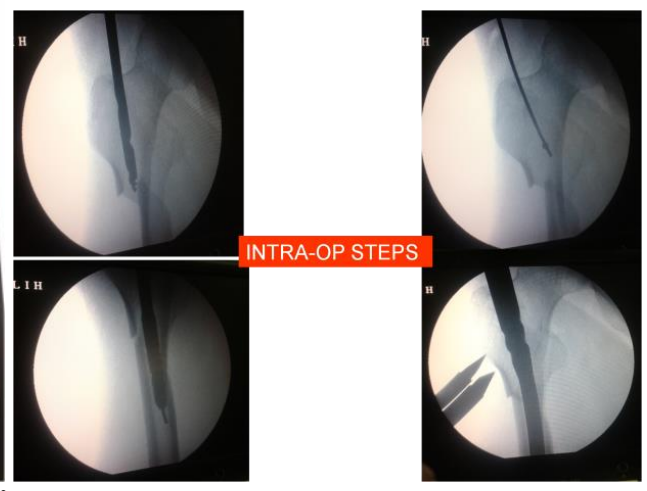

intra op 


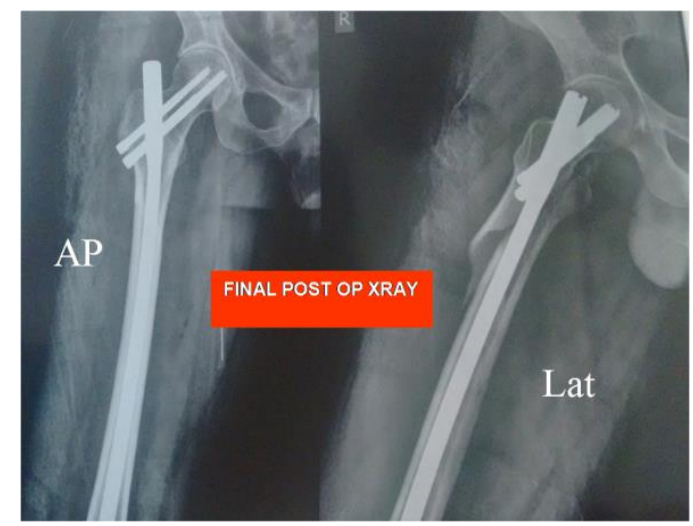

Immed post op X-ray

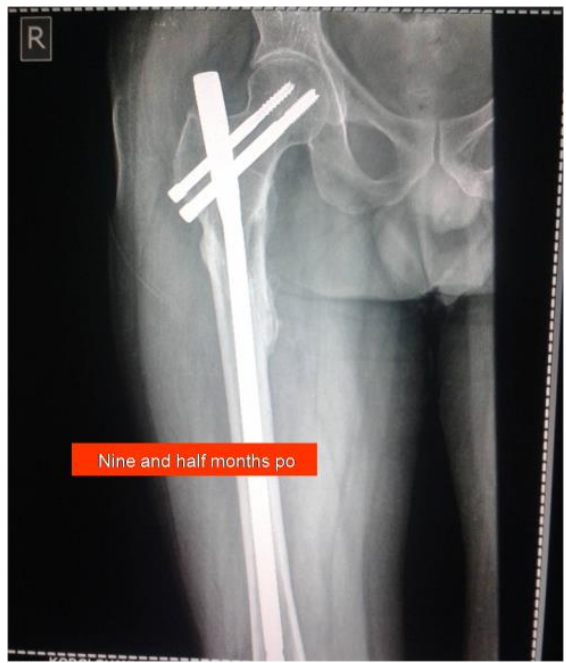

Nine months follow up full union

\section{Results}

In the present study, age group of patients was $25 \mathrm{yrs}$ to $74 \mathrm{yrs}$. Male patients $62(83.7 \%)$ were more than female patients $12(16.3 \%)$

Blood Loss: An average of less than $150 \mathrm{ml}$ in closed surgery. And about $350 \mathrm{ml}$ in open reduction cases was used.

Operating Time: Duration of surgery was considered from time of incision to skin closure. It was 60 minutes to 120 minutes mean operating time 80 minutes.

Subtrochanteric comminuted fractures lead to blood loss, so blood transfusion pre op, intra op, and post operatively were transfused as per case demanded. Blood transfusion varied from one to four per case or more in polytraumatized patients.

Long PFN in comminuted subtrochanteric fractures of femur in lateral position succeed in 69 cases $(93 \%)$.

Five cases required open reduction by exposing the fracture site because of soft tissue entrapment at fracture ends which was cleared and negotiated later.

Four cases were grade two compound with anterolateral wound which was primarily debrided and sutured well and they healed up well. In these compound fractures, this rent helped us to get proper reduction and passing nail by manipulating and

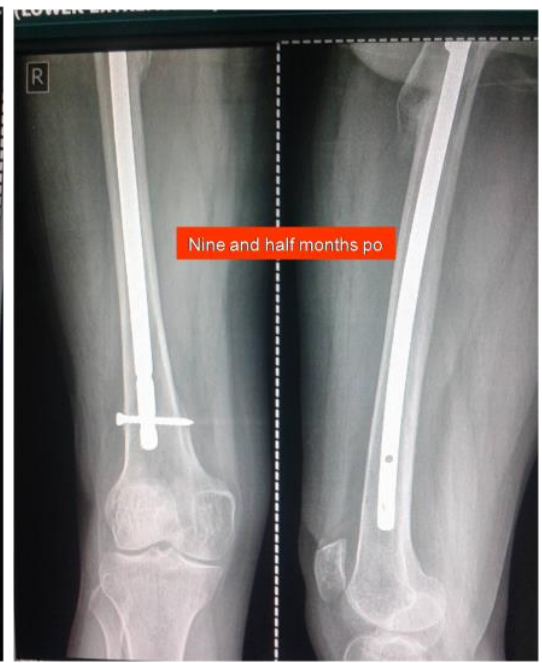

handling the bony segments. There was no any signs of infection later in the follow up.

Segmental Fracture: subtrochanteric and associated shaft fracture femur TWO cases.

The quality of reduction was determined assessing displacement of main fracture fragments on post operative X-rays. Reductions were considered "Anatomic" if there was less than $2 \mathrm{~mm}$ of displacement. Reductions with displacements of 2 to 5 $\mathrm{mm}$ were termed "Near Anatomic". "Anatomic reductions" were achieved in simple transverse or short oblique non comminuted subtrochanteric fractures, whereas "near anatomic reductions" were achieved in comminuted subtrochanteric fractures. In one or the other case though there was large displacement of fragment in one view they ultimately consolidated with parent bone in due course of follow up.

Shortening of limb was anticipated in few comminuted cases with mean shortening of $1.8 \mathrm{cms}$ (range $1 \mathrm{~cm}$ to $2.8 \mathrm{~mm}$ ) with mild $5-7^{*}$ varus deformity. Those with shortening did not require any shoe raise and managed well in all activities of daily living.

Union time varied from 16 weeks in simple fractures to 26 weeks in comminuted fractures. Clinical function of hip and knee was excellent with full range of movements. Few elderly patients with pre existing 
osteoarthritis of knee had terminal restriction of flexion of knee and had no correlation to operative technique.

\section{Discussion}

Davis et al. in 1969 used the lateral position as a facilitated position for reduction and exposure for the first time in intertrochanteric and subtrochanteric fractures of the femur, Ozkan et al. used this position in proximal femoral nailing in 2010 and Connelly et al. in complex proximal femur locked plating in $2012 .^{7-9}$ In our study minimal surgical incision nice reduction and alignment of fracture in lateral position, hemostasis, and decreased surgery time and less radiation of C-arm was observed. However, lack of a control group made the comparisons impossible.

Problems in Supine Position on A Fracture Table: In subtrochanteric, and high subtrochanteric femur fractures, proximal segment is flexed \& abducted due to pull of strong muscles around the hip. (Fig. 10)
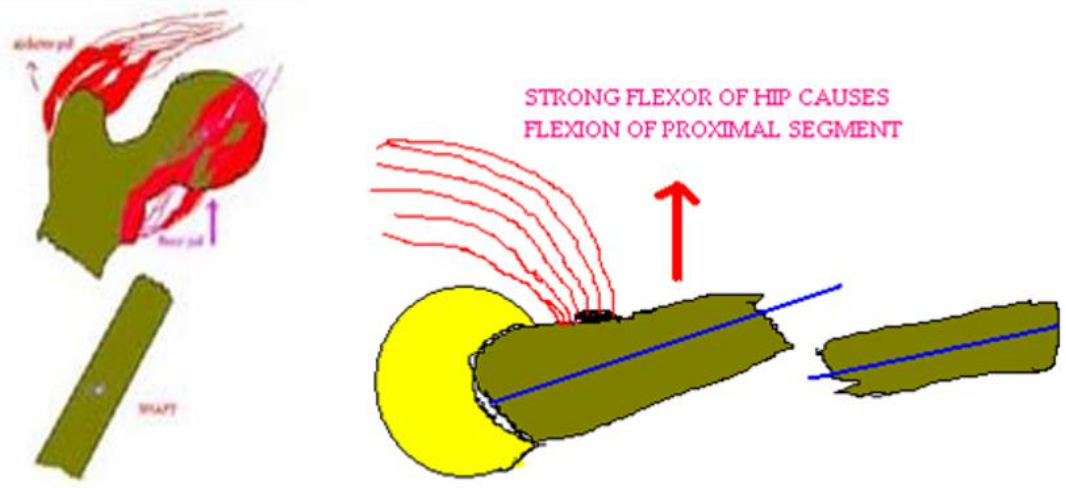

Fig. 10: Muscle forces around hip with displacement and angulation of segments

Obesity/pendulous sagging abdomen makes entry of awl in proximal femur (pyriformis fossa) guide wire passing reaming difficult and obstructs insertion of PFN nail and jig assembly. Osteoarthritic knees with flexion deformity of knee causes sagging of shaft portion of femur making entry difficult as well as negotiation of guide wire reamer and nail in distal segment. (Fig. 11)

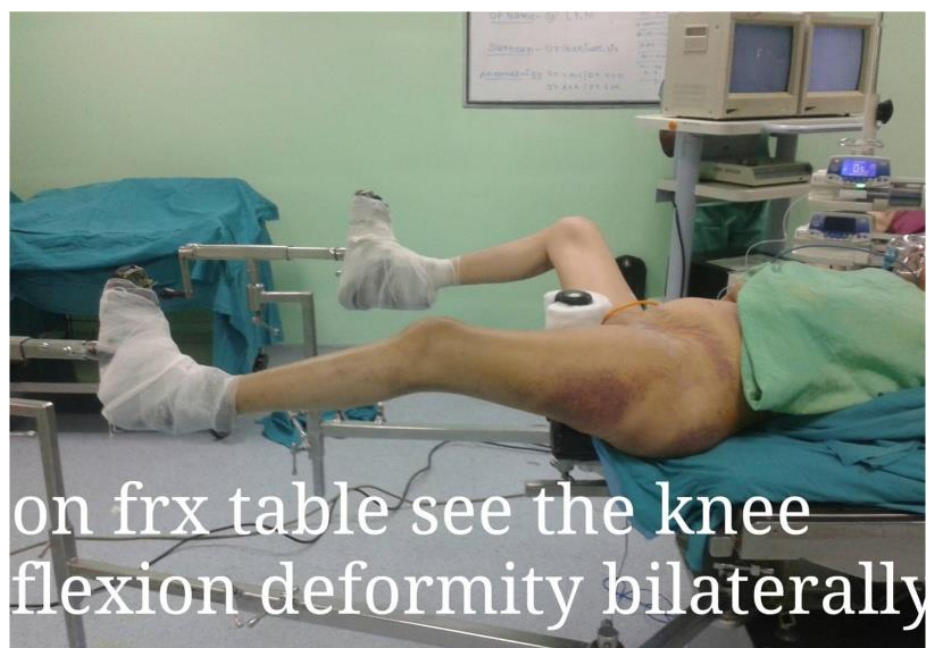

Fig. 11

$\mathrm{C}$-arm adjustments have to be made number of times to check in AP/Lateral views by moving the heavy body of $\mathrm{C}$-arm machine. As the operative thigh has to be adducted for having a good entry in pyriformis fossa there is limited and constrained space for moving $\mathrm{c}$ arm and adjusting the $\mathrm{c}$ arm between the two legs of the patient. Lateral view of neck is difficult to visualize with adducted operative limb.

As the foot of patient is tied at the footplate of fracture table, manipulation of displaced small and large comminuted fragments and segment is difficult. In case of difficulty if fracture needs open reduction the soft tissues and muscles are severely stretched in traction on fracture table not allowing the surgeon to manipulate the segments easily. Secondly haemostasis achievement also becomes difficult.

Advantages in Lateral Position: In lateral position all muscles around hip are relaxed. Affected limb is freely draped to have free movement. Distal segment can be mobilized to align with proximal segment freely with gentle longitudinal traction holding the knee or leg. 
In lateral position proximal segment can be manually pushed to get good adduction \& have a easy access in pyriformis fossa (entry). Further procedures i.e. gw insertion, negotiation of distal canal for reaming \& nail insertion becomes easy. C-arm remains stationary showing AP view as patient is lying in lateral position. C-arm adjustment is not required to view AP/Lateral views. Visualisation of Lateral view of neck Femur is possible by just flexing \& abducting and mild external rotation of limb to see guide wire /proximal screws in neck/head without moving the heavy machine body.

In case the operating theatre is small the $\mathrm{C}$ arm along with fracture table is occupying much space so in such situation, the $\mathrm{C}$ arm can be adjusted in Scorpion flangs position such that the $\mathrm{C}$ arm is horizontally aligned from foot end and can be adjusted accordingly. [Fig. 12]

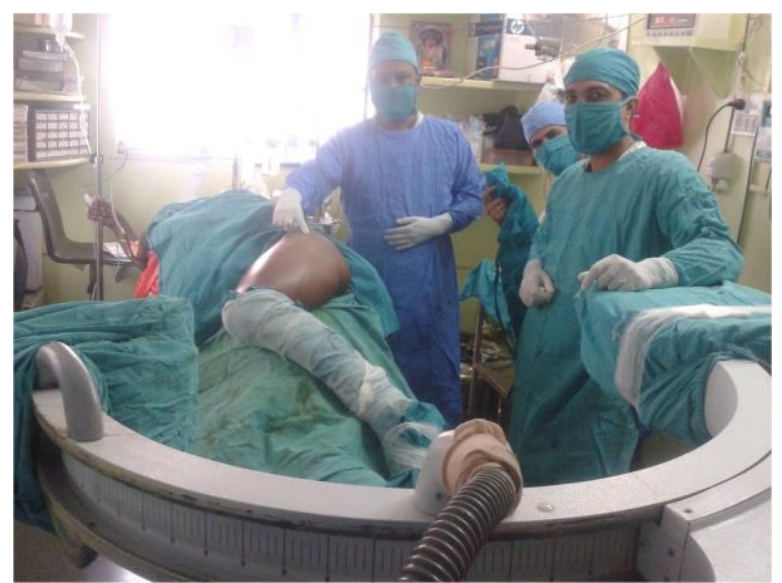

Fig. 12: Scorpion flang position of $\mathrm{C}$ arm in smaller space operation theatre

The possibility of distal interlocking with distraction at fracture site is more in operating on fracture table with fixed traction, where as this possibility is not present as all muscles are relaxed in lateral position.

Muscular forces around hip in sagittal plane (Hip flexors and extensors) are more effectively neutralized in lateral position where as the coronal plane forces (Abductors and Adductors) are easily neutralized with a firm pillow between two legs in lateral position. ${ }^{9}$

In present study, average of less than $150 \mathrm{ml}$ in closed surgery and about $350 \mathrm{ml}$ in open reduction cases, blood lost. It was showed in this meta-analysis that surgery in lateral decubitus was marginally associated with less blood loss compared with surgery in supine position. ${ }^{15}$

There were some limitations in our study, firstly, lack of a control group made the comparisons of minimal surgical incision reduction and alignment of fracture in lateral position, hemostasis, and decreased surgery time and less radiation of $\mathrm{C}$-arm are impossible. Secondly, we did not use an accepted outcome measure instrument for evaluation of the patients' ability to squat and walk with no limp for assessing outcome was also a crude method. But assessment for bony union with serial radiographs in our study was acceptable.

\section{Conclusion}

Multiple changing of $\mathrm{C}$ arm positions during surgery on fracture table between two legs on fracture table and very narrow space for moving $\mathrm{C}$ arm in attempt to visualize the guide wire and screws in head neck of femur, lead to delaying of completing the surgery and bleeding with increased anaesthesia and operative time and wound exposure leading to infection and increased exposure to radiation of $\mathrm{C}$ arm. In our study the frog leg view or lateral view of neck could be visualized by just gentle flexing and abducting with mild external rotation of operative femur without repositioning $\mathrm{C}$-arm in different positions.

Operating subtrochanteric comminuted fracture femur in lateral position on an ordinary top table is much easier than on fracture table.

Reduction and fixation of proximal femoral fractures in the lateral position with fluoroscopy or portable radiography in the anteroposterior view for small set ups and rural hospitals that lack a fracture table or advanced fluoroscopic devices may be executable and probably safe.

\section{References}

1. Nork SE, Reilly MC. Skeletal Trauma. 4th ed. Philadelphia, PA: WB Saunders; 2008. Subtrochanteric fractures of the femur; pp. 1977-2034.

2. Egol K, Koval KJ, Zuckerman JD. Handbook of fractures. Lippincott Williams \& Wilkins; 2010.

3. Arch Bone Jt Surg. 2014;2(3):168-173

4. Smith-Petersen MN, Cave EF, Vangorder GW. Intracapsular fractures of the neck of the femur: treatment by internal fixation. Archives of Surgery. 1931;23(5):715.

5. Caldwell JA. Subtrochanteric fractures of the femur: An operative approach for open fixation. The American Journal of Surgery. 1943;59(2):370-82.

6. Horwitz T. The posterolateral approach in the surgical management of basilar neck, intertrochanteric and subtrochanteric fractures of the femur; a report of its use in 36 acute fractures. Surgery, gynecology \& obstetrics. 1952;95(1):45-50.

7. Davis PH, Frymoyer JW. The lateral position in the surgical management of intertrochanteric and subtrochanteric fractures of the femur. J Bone Joint Surg Am. 1969;51(6):1128-34.

8. Ozkan K, Cift H, Akan K, Sahin A, Eceviz E, Ugutmen E. Proximal femoral nailing without a fracture table. European Journal of Orthopaedic Surgery \& Traumatology. 2010;20(3):229-31.

9. Camille L. Connelly. J. Orthop Trauma. 2012;26(4):252257.

10. Anatomic Reduction and Locking Plate Neutralization: A Technical Trick. Journal of Orthopaedic Trauma. 2012;26(4):252.

11. Callanan I, Choudhry V, Smith H. Perineal sloughing as a result of pressure necrosis from the traction post during prolonged bilateral femoral nailing. Injury. 1994;25(7):472 
12. Brumback RJ, Ellison TS, Molligan H, Molligan DJ, Mahaffey S, Schmidhauser C. Pudendal nerve palsy complicating intramedullary nailing of the femur. $J$ Bone Joint Surg Am. 1992;74(10):1450-5.

13. Carr JB, Williams D, Richards M. Lateral decubitus positioning for intramedullary nailing of the femur without use of fracture table Orthopaedics. 2009;32(10).

14. Winquist R, Hansen S, Clawson D. Closed intramedullary nailing of femoral fractures. A report of five. J Bone Joint Surg Am. 1984;66:529-39.

15. Müller M. CCF-Comprehensive Classification of Fractures I \& II. MEM üller Foundation. Bern: MAO/ ASIF Documentation Center; 1996.16. Lin J.
Encouraging results of treating femoral trochanteric fracture.

16. Liu. A Meta-analysis Comparing Lateral Decubitus with Supine Position for Surgery for Intertrochanteric Fractures. J Arthritis. 2016;5:4.

How to cite this article: Shashikant B. Ganjale. Long PFN nailing in comminuted high subtrochanteric fractures femur in lateral position on ordinary table. Indian $\mathrm{J}$ Orthop Surg. 2018;4(3):273-281. 